\title{
On a $C^{*}$-Algebra Approach to Phase Transition in the Two-Dimensional Ising Model
}

\author{
Huzihiro Araki and David E. Evans* \\ Research Institute for Mathematical Sciences. Kyoto University, Kyoto 606, Japan
}

\begin{abstract}
We investigate the state on the $C^{*}$-algebra of Pauli spins on a onedimensional lattice (infinitely extended in both directions) which gives rise to the thermodynamic limit of the Gibbs ensemble in the two-dimensional Ising model (with nearest neighbour interaction). It is shown that the representation of the Pauli spin algebra associated with the state is factorial above and at the known critical temperature, while it has a two-dimensional center below the critical temperature. As a technical tool, we derive a general criterion for a state of the Pauli spin algebra corresponding to a Fock state of the Fermion algebra to be primary. We also show that restrictions of two quasifree states of the Fermion algebra to its even part are equivalent if and only if the projection operators $E_{1}$ and $E_{2}$ (on the direct sum of two copies of the basic Hilbert space) satisfy the following two conditions: (1) $E_{1}-E_{2}$ is in the Hilbert-Schmidt class, (2) $E_{1} \wedge(1$ $\left.-E_{2}\right)$ has an even dimension, where the even-oddness of $\operatorname{dim} E_{1} \wedge\left(\mathbf{1}-E_{2}\right)$ is called $\mathbb{Z}_{2}$-index of $E_{1}$ and $E_{2}$ and is continuous in $E_{1}$ and $E_{2}$ relative to the norm topology.
\end{abstract}

\section{Main Results}

We consider the two-dimensional Ising model with the Hamiltonian (with the free boundary condition)

$$
H^{L M}(\xi)=-\left(\sum_{i=-L}^{L-1} \sum_{j=-M}^{M} J_{1} \xi_{i j} \xi_{i+1, j}+\sum_{i=-L}^{L} \sum_{j=-M}^{M-1} J_{2} \xi_{i j} \xi_{i, j+1}\right),
$$

where $\xi_{i j}= \pm 1$ is the (classical) spin at the lattice site $(i, j) \in \mathbb{Z}^{2}$, and $J_{1}$ and $J_{2}$ are positive constants. We are interested in the thermodynamic limit $(L, M \rightarrow \infty)$ of the Gibbs ensemble average

$$
\begin{aligned}
\langle F\rangle_{L M} & =Z_{L M}^{-1} \sum_{\xi} F(\xi) e^{-\beta H^{L M}(\xi)}, \\
Z_{L M} & =\sum_{\xi} e^{-\beta H^{L M}(\xi)},
\end{aligned}
$$

* On leave from Mathematics Institute, University of Warwick, Coventry, CV4 7AL, England 
where the sum is over all configurations $\xi=\left\{\xi_{i j}\right\}, \beta \geqq 0$, and $F$ is a function of $\xi_{i j},|i| \leqq l,|j| \leqq m$ for some $l \leqq L$ and $m \leqq M$.

There is the following transfer matrix method [9] of expressing it in terms of a state on the $C^{*}$-algebra $\mathfrak{U}^{P}$ generated by Pauli spin matrices $\sigma_{x}^{(i)}, \sigma_{y}^{(i)}, \sigma_{z}^{(i)}$ on sites $i \in \mathbb{Z}$ of the one-dimensional lattice $\mathbb{Z}$.

By identifying a function of $\xi_{j}= \pm 1$ and $\xi_{j}^{\prime}= \pm 1$ as the $\left(\xi_{j}, \xi_{j}^{\prime}\right)$ matrix element of a linear combination of $2 \times 2$ matrices $1, \sigma_{x}^{(j)}=\left(\begin{array}{rr}1 & 0 \\ 0 & -1\end{array}\right), \sigma_{y}^{(j)}=\left(\begin{array}{rr}0 & i \\ -i & 0\end{array}\right)$ and $\sigma_{z}^{(j)}=\left(\begin{array}{ll}0 & 1 \\ 1 & 0\end{array}\right)$, we have the identification

$$
\begin{aligned}
\left(T_{M}\right)_{\xi, \xi^{\prime}}= & \exp \left\{\frac{K_{2}}{2} \sum_{j=-M}^{M-1}\left(\xi_{j} \xi_{j+1}+\xi_{j}^{\prime} \xi_{j+1}^{\prime}\right)+K_{1} \sum_{j=-M}^{M} \xi_{j} \xi_{j}^{\prime}\right\}, \\
T_{M}= & \left(2 \sinh 2 K_{1}\right)^{M+(1 / 2)} \exp \left\{\frac{K_{2}}{2} \sum_{j=-M}^{M-1} \sigma_{x}^{(j)} \sigma_{x}^{(j+1)}\right\} \\
& \times \exp \left\{K_{1}^{*} \sum_{j=-M}^{M} \sigma_{z}^{(j)}\right\} \exp \left\{\frac{K_{2}}{2} \sum_{j=-M}^{M-1} \sigma_{x}^{(j)} \sigma_{x}^{(j+1)}\right\},
\end{aligned}
$$

where $K_{j}=\beta J_{i}(j=1,2)$,

$$
K_{1}^{*}=(1 / 2) \log \left\{\operatorname{coth} K_{1}\right\}
$$

and $\xi=\left(\xi_{j}\right), \xi^{\prime}=\left(\xi_{j}^{\prime}\right)$. Then

$$
\begin{aligned}
Z_{L M} & =\left\|\left(T_{M}\right)^{L} \Omega_{M}\right\|^{2}, \\
\Omega_{M}(\xi) & =\exp \left\{\frac{K_{2}}{2} \sum_{j=-M}^{M-1} \xi_{j} \xi_{j+1}\right\}, \\
\langle F\rangle_{L M} & =\left(T_{M}^{L} \Omega_{M}, F_{\beta M} T_{M}^{L} \Omega_{M}\right) / Z_{L M},
\end{aligned}
$$

where $F_{\beta M} \in \mathfrak{U}^{P}$. (For example, if $F=\prod_{i=-l}^{l} F_{i}$ with $F_{i}=F_{i}\left(\xi_{i,-m} \ldots \xi_{i m}\right)$, then $F_{\beta M}=T_{M}^{-l} \hat{F}_{-l} T_{M} \hat{F}_{-l+1} \ldots T_{M} \hat{F}_{l} T_{M}^{-l}$ with $\hat{F}_{i}^{i=-l}=F_{i}\left(\sigma_{x}^{(-m)} \ldots \sigma_{x}^{m}\right)$.)

The $L \rightarrow \infty$ limit selects the unique unit eigenvector $\Omega^{M}=\Omega^{M}(\xi)\left(\Omega^{M}(\xi)>0\right)$ belonging to the largest eigenvalue of $T_{M}$.

$$
\lim _{L \rightarrow \infty}\langle F\rangle_{L M}=\left(\Omega^{M}, F_{\beta M} \Omega^{M}\right)
$$

In $M \rightarrow \infty$ limit, $F_{\beta M}$ is known to converge to an element $F_{\beta}$ of $\mathfrak{U}^{P}$ (it is $\alpha_{i l}^{M}\left(\hat{F}_{-l}\right) \ldots \alpha_{i}^{M}\left(\hat{F}_{-1}\right) \hat{F}_{0} \alpha_{-i}^{M}\left(\hat{F}_{1}\right) \ldots \alpha_{-i l}^{M}\left(\hat{F}_{l}\right)$ for $\alpha_{i l}^{M}(x)=T_{M}^{-l} x T_{M}^{l}$, which converges for all integers $l$, see [2]), whilst the limit of the vector state by $\Omega^{M}$

$$
\varphi_{\beta}(a)=\lim _{M \rightarrow \infty}\left(\Omega^{M}, a \Omega^{M}\right)
$$


will be explicitly given in Sect. 2 , so that

$$
\varphi_{\beta}\left(F_{\beta}\right)=\lim _{M \rightarrow \infty} \lim _{L \rightarrow \infty}\langle F\rangle_{L M} .
$$

Our main result is the following:

Theorem 1. The cyclic representation of $\mathfrak{Q}^{P}$ associated with $\varphi_{\beta}$ is factorial if $0 \leqq$ $\beta \leqq \beta_{c}$, whilst it is non-factorial with 2-dimensional center (for the weak closure) if $\beta>\beta_{c}$, where $\beta_{c}$ is the unique value of $\beta>0$ for which $K_{1}^{*}=K_{2}$.

The two-dimensional Ising model with nearest neighbour interactions was shown to have a phase transition in [13]. There is a critical (inverse) temperature $\beta_{c}$ such that the spontaneous magnetisation $m^{*}=0$ for $\beta<\beta_{c}$, and $m^{*} \neq 0$ for $\beta>\beta_{c}$. Moreover for $\beta<\beta_{c}$, a unique equilibrium state exists, whilst for $\beta>\beta_{c}$, there are exactly two distinct extremal equilibrium states $[1,5]$. Schultz, Mattis and Lieb [15] reformulated the Onsager-Kaufman transfer matrix treatment [7, 8] using a Fermion algebra $\mathfrak{Q}^{F}$. Now the even subalgebras of $\mathfrak{U}^{P}$ and $\mathfrak{U}^{F}$ are canonically isomorphic, even though the algebras $\mathfrak{A}^{P}$ and $\mathfrak{U}^{F}$ themselves are not (for the system infinitely extended to all directions), and so one has a correspondence between even states on the Pauli spin algebra $\mathfrak{U}^{P}$ and even states on the Fermion algebra $\mathfrak{A}^{F}$. For a two dimensional lattice, infinitely extended in all directions, the Gibbs state in the thermodynamic limit induces a pure (hence primary) Fock state on the Fermion algebra $\mathfrak{Q}^{F}[14,11,16]$. In $[11]$ Lewis and Sisson discussed how the phase transition manifests itself by a jump in the index of a Fredholm operator associated with the Fock state. Subsequently, Lewis and Winnink [12] showed that the phase transition also reveals itself in the restricted state on the Fermion algebra $\mathfrak{U}^{F}([1, \infty))$ of a halfline (regarded as a subalgebra of $\mathfrak{Q}^{F}$ ). The restricted state is a non-Fock quasi-free state. It is primary for $\beta<\beta_{c}$, and non-primary for $\beta>\beta_{c}$. Again this involves the computation of an index $(\bmod 2)$ of a Fred holm operator. For a half lattice, the Pauli spin algebra $\mathfrak{A}^{P}([1, \infty))$ is canonically isomorphic to $\mathfrak{A}^{F}([1, \infty))$, and Kuik in [10] showed that (for periodic boundary conditions) the thermodynamic limit of the Gibbs state induces precisely the above restricted state on $\mathfrak{U}^{P}([1, \infty))$. Hence for a half lattice, the manifestation of the phase transition is apparent.

In the present case of a two dimensional lattice, infinitely extended to all directions (in contrast to a half lattice), the state for the Fermion algebra is pure (Fock state) for all $\beta$, but our Theorem 1 shows that the phase transition manifests itself in the state for the Pauli spin algebra, which is primary for $0 \leqq \beta \leqq \beta_{c}$ and nonprimary for $\beta>\beta_{c}$.

\section{Correspondence of Pauli and Fermion Algebras}

Following [4], we consider the $C^{*}$-algebra $\mathfrak{A}^{F}$ (the Fermion algebra) generated by annihilation and creation operators $c_{i}$ and $c_{i}^{*}(i \in \mathbb{Z})$ satisfying the canonical anticommutation relations (CAR's):

$$
\left[c_{i}, c_{j}\right]_{+}=\left[c_{i}^{*}, c_{j}^{*}\right]_{+}=0,\left[c_{i}, c_{j}^{*}\right]_{+}=\delta_{i j} \mathbf{1} .
$$


Defining the automorphism $\Theta_{\text {_ of }} \mathfrak{A}^{F}$ satisfying

$$
\Theta_{-} c_{i}=\left\{\begin{aligned}
c_{i} & \text { if } i \geqq 1 \\
-c_{i} & \text { if } i \leqq 0,
\end{aligned}\right.
$$

we construct the crossed product $\hat{\mathfrak{U}}=\mathfrak{U}^{F} \times_{\Theta} \mathbb{Z}_{2}$, which is generated by $\mathfrak{U}^{F}$, and $T \in \hat{\mathfrak{A}}$ satisfying

$$
\begin{aligned}
& T^{2}=\mathbf{1}, T^{*}=T, \\
& T a=\Theta_{-}(a) T \quad\left(a \in \mathfrak{U}^{F}\right) .
\end{aligned}
$$

The Pauli spin algebra $\mathfrak{Q}^{P}$ can be identified with a $C^{*}$-subalgebra of $\hat{\mathfrak{A}}$ generated by

$$
\begin{aligned}
& \sigma_{z}^{(j)}=2 c_{j}^{*} c_{j}-1, \\
& \sigma_{x}^{(j)}=T S_{j}\left(c_{j}+c_{j}^{*}\right), \quad \sigma_{y}^{(j)}=T S_{j} i\left(c_{j}-c_{j}^{*}\right), \\
& S_{j}= \begin{cases}\prod_{k=1}^{j-1} \sigma_{z}^{(k)} & \text { if } j>1, \\
1 & \text { if } j=1, \\
\prod_{k=0}^{j} \sigma_{z}^{(k)} & \text { if } j<1 .\end{cases}
\end{aligned}
$$

Let $\Theta$ be the automorphism of $\hat{\mathfrak{U}}$ satisfying

$$
\Theta\left(c_{i}\right)=-c_{i}, \quad \Theta(T)=T .
$$

We have

$$
\Theta\left(\sigma_{z}^{(j)}\right)=\sigma_{z}^{(j)}, \Theta\left(\sigma_{x}^{(j)}\right)=-\sigma_{x}^{(j)}, \Theta\left(\sigma_{y}^{(j)}\right)=-\sigma_{y}^{(j)} .
$$

The Fermion algebra $\mathfrak{A}^{F}$ is split into even and odd parts:

$$
\mathfrak{U}^{F}=\mathfrak{U}_{+}^{F}+\mathfrak{U}_{-}^{F}, \mathfrak{U}_{ \pm}^{F}=\left\{a \in \mathfrak{U}^{F}: \Theta(a)= \pm a\right\} .
$$

Accordingly

$$
\begin{aligned}
\hat{\mathfrak{U}} & =\sum_{\sigma, \sigma^{\prime}= \pm} \hat{\mathfrak{U}}_{\sigma \sigma^{\prime}} \supset \mathfrak{U}^{P}=\mathfrak{U}_{+}^{P}+\mathfrak{U}_{-}^{P}, \\
\hat{\mathfrak{U}}_{\sigma+} & =\mathfrak{U}_{\sigma}^{F}, \hat{\mathfrak{U}}_{\sigma-}=T \mathfrak{U}_{\sigma}^{F}(\sigma= \pm), \\
\mathfrak{U}_{+}^{P} & =\hat{\mathfrak{U}}_{++}=\mathfrak{U}_{+}^{F}, \mathfrak{U}_{-}^{P}=\hat{\mathfrak{U}}_{--}=T \mathfrak{U}_{-}^{F} .
\end{aligned}
$$

By the relation (2.5) and

$$
\sigma_{x}^{(i)} \sigma_{x}^{(i+1)}=\left(c_{i}-c_{i}^{*}\right)\left(c_{i+1}+c_{i+1}^{*}\right),
$$

$T_{M}$ is given, up to a positive coefficient, by

$$
T_{M}^{\prime}=e^{\left(K_{2} / 2\right)\left(B^{M}, H_{2} B^{M}\right)} e^{K_{1}^{*}\left(B^{M}, H_{1} B^{M}\right)} e^{\left(K_{2} / 2\right)\left(B^{M}, H_{2} B^{M}\right)},
$$

where

$$
H_{2}=\frac{1}{2}\left(\begin{array}{cc}
-\left(U^{*}+U\right) & U^{*}-U \\
U-U^{*} & U^{*}+U
\end{array}\right), \quad H_{1}=\left(\begin{array}{rr}
1 & 0 \\
0 & -1
\end{array}\right)
$$




$$
(U f)_{k}=f_{k+1},\left(U^{*} f\right)_{k}=f_{k-1}\left(f=\left(f_{k}\right) \in l_{2}(\mathbb{Z})\right),
$$

and we have used the following notation of [3]:

$$
\begin{aligned}
B^{M}(h) & \equiv \sum_{j=-M}^{M}\left(c_{j}^{*} f_{j}+c_{j} g_{j}\right)\left(h=\left(\begin{array}{l}
f \\
g
\end{array}\right), f=\left(f_{j}\right), g=\left(g_{j}\right)\right), \\
\left(B^{M}, H B^{M}\right) & =\sum_{m n} B^{M}\left(e_{m}\right)\left(e_{m}, H e_{n}\right) B^{M}\left(e_{n}\right)^{*},
\end{aligned}
$$

for an orthonormal basis $\left\{e_{n}\right\}$ of

$$
\mathscr{K}_{M}=\left\{\left(\begin{array}{l}
f \\
g
\end{array}\right) \in l_{2} \oplus l_{2} ; f_{j}=g_{j}=0 \text { if }|j|>M\right\} .
$$

We note that $H_{j}(j=1,2)$ satisfy

$$
H_{j}^{*}=H_{j}, \quad \Gamma H_{j}+H_{j} \Gamma=0,
$$

where $\Gamma\left(\begin{array}{c}f \\ g^{*}\end{array}\right) \equiv\left(\begin{array}{c}g \\ f^{*}\end{array}\right)$ and $B^{M}(h)^{*}=B^{M}(\Gamma h)$.

Let $H^{M}$ be the unique selfadjoint operator satisfying

$$
e^{2 H^{M}}=e^{K_{2} H_{2}^{M}} e^{2 K_{1}^{*} H_{1}^{M}} e^{K_{2} H_{2}^{M}},
$$

where $H_{j}^{M}$ is the restriction of $H_{j}$ to $l_{2}(\{-M, \ldots, M\})$. Due to $\operatorname{tr} \sigma_{x}^{(j)}=\operatorname{tr} \sigma_{z}^{(j)}=0$, we have det $e^{H^{M}}=1$. Since the right-hand side of (2.22) is analytic in $K_{1}^{*}$ and $K_{2}$, so is $H$ for all real $K_{1}^{*}$ and $K_{2}$. By repeated use of (8.48) in [3] (also see the definition (8.23)), we have

$$
e^{\left(B^{M}, H^{M} B^{M}\right)}=T_{M}^{\prime},
$$

where the possible sign \pm 1 multiplying $T_{M}^{\prime}$ (according to the use of (8.48)) is +1 because it is +1 for $K_{1}^{*}=K_{2}=0$, and both sides of (2.23) are real analytic in $K_{1}^{*}$ and $K_{2}$.

\section{The State $\varphi_{\beta}$}

Let $E_{-}^{M}, E_{0}^{M}$ and $E_{+}^{M}$ be the spectral projection of $H^{M}$ for $(-\infty, 0),\{0\}$ and $(0, \infty)$. Due to (2.21), $\Gamma E_{ \pm}^{M} \Gamma=E_{\mp}^{M},\left[\Gamma, E_{0}\right]=0$. Let $\left\{e_{v}\right\}$ be a complete orthonormal basis of $E_{+}^{M} \mathscr{K}_{M}$, satisfying $H^{M} e_{v}=\lambda_{v} e_{v}\left(\lambda_{v}>0\right)$, and set $b_{v}=B\left(e_{v}\right)$. Due to $B(h)^{*}=B(\Gamma h)$ and

$$
\left[B\left(h_{1}\right)^{*}, B\left(h_{2}\right)\right]_{+}=\left(h_{1}, h_{2}\right) \mathbf{1},
$$

$\left\{b_{v}\right\}$ satisfy CAR's among themselves. (Note that $\left\{\Gamma e_{v}\right\}$ is an orthonormal basis of $E_{-}^{M} \mathscr{K}_{M}$ satisfying $H^{M} \Gamma e_{v}=-\lambda_{v} \Gamma e_{v}$.) Furthermore

$$
\left(B^{M}, H^{M} B^{M}\right)=\sum \lambda_{v}\left(b_{v} b_{v}^{*}-b_{v}^{*} b_{v}\right)=\sum_{v} \lambda_{v}\left(1-2 b_{v}^{*} b_{v}\right) .
$$

Therefore the eigensubspace of $\left(B^{M}, H^{M} B^{M}\right)$ belonging to the largest eigenvalue consists of vectors $\Omega$ (in $l^{2}\left(\{1,-1\}^{2 M+1}\right)$ ) satisfying $b_{v} \Omega=0$ for all $v$ and has the dimension $2^{\operatorname{dim} E_{0}^{M}}$. 
For finite $K_{1}^{*}$, all matrix elements of $e^{K_{1}^{*}\left(B^{M}, H_{1} B^{M}\right)}$ are strictly positive and hence the same is true for $T_{M}$. By the Perron-Frobenius theorem, the largest eigenvalue of $T_{M}$ is non-degenerate as is claimed in Sect. 1.

In the finite dimensional case, one can view the Pauli algebra $\mathfrak{U}^{{ }^{P M}}$ generated by $\sigma_{x}^{(j)}, \sigma_{y}^{(j)}, \sigma_{z}^{(j)},|j| \leqq M$ as the Fermion algebra generated by $\bar{c}_{j}$ and $\bar{c}_{j}^{*}$ related to $\sigma^{\prime}$ 's by $(2.5) \sim(2.7)\left(c\right.$ 's replaced by $\vec{c}$ 's) with the difference that $T=\prod_{j=0}^{-M} \sigma_{z}^{(j)}=\prod_{j=0}^{-M}\left(2 \bar{c}_{j} \bar{c}_{j}^{*}-\mathbf{1}\right)$. Still we obtain the same expression for $T_{M}$, and hence we conclude that the largest eigenvalue of $\left(B^{M}, H^{M} B^{M}\right)$ must be non-degenerate, which implies $E_{0}^{M}=0$ and

$$
1-E_{-}^{M}=E_{+}^{M}=\Gamma E_{-}^{M} \Gamma \text {. }
$$

We now see that the vector state $\varphi_{M}^{+}(a)=\left(\Omega^{M}, a \Omega^{M}\right)$ of $\mathfrak{U}_{+}^{P M}$ has the property that

$$
\varphi_{M}^{+}\left(B^{M}(h)^{*} B^{M}(h)\right)=0 \quad \text { if } h \in E_{+}^{M} \mathscr{K}_{M} .
$$

Any state $\varphi$ on $\mathfrak{I}_{+}^{P M}$ has a (unique) extension to a $\Theta$-invariant state $\hat{\varphi}$ on the Fermion algebra $\mathfrak{U}^{F M}$ (generated by $c_{j}$ and $c_{j}^{*},|j| \leqq M$ ) by $\hat{\varphi}(a)=\varphi\left(a_{+}\right), a_{ \pm}=(a \pm \Theta(a)) / 2$, $a \in \mathfrak{A}^{F M}$. The extended state $\hat{\varphi}$ must be the Fock state, for which $\vec{b}_{v}$ and $b_{v}^{*}$ act as annihilation and creation operators due to (3.4). Such a state has been denoted as $\varphi_{P}$ in [3] with $P=E_{-}^{M}$ satisfying property (3.3) for a "basis projection."

In the limit of $M \rightarrow \infty, U^{M}$ and $\left(U^{*}\right)^{M}$ (restrictions of $U$ and $U^{*}$ to the subspace $\mathscr{K}_{M}$, which are no longer unitary) tend strongly to $U$ and $U^{*}$. Hence $H_{j}^{M}$ tends to $H_{j}(j=1,2)$ and $H^{M}$ to $H$ defined by

$$
e^{2 H}=e^{K_{2} H_{2}} e^{2 K_{1}^{*} H_{1}} e^{K_{2} H_{2}}, \quad H^{*}=H .
$$

Due to the property $\Gamma H_{j} \Gamma=-H_{j}, H$ also satisfies $\Gamma H \Gamma=-H$.

We need the following result:

Lemma 3.1. $H$ does not have a point spectrum at 0 .

Before going into its proof, we describe its consequence. Since $H$ does not have the point spectrum at 0 , Lemma 3.3 at the end of the section implies

$$
\lim _{M \rightarrow \infty} E_{ \pm}^{M}=E_{ \pm}
$$

where $E_{-}$and $E_{+}$are spectral projections of $H$ for $(-\infty, 0)$ and $(0, \infty)$. This means that the limit of $\varphi_{M}^{+}$as $M \rightarrow \infty$ is the restriction of the Fock state $\varphi_{E_{-}}$on $\mathfrak{U}^{F}$ to $\mathfrak{U}_{+}^{F}=\mathfrak{U}_{+}^{P}$.

The automorphism $\Theta$ on $\mathfrak{A}^{P M}$ can be implemented by a unitary operator $U(\Theta)=\prod_{j=-M}^{M} \sigma_{z}^{(j)}$. By $\Theta$-invariance of $H^{M}, \Omega^{M}$ is $U(\Theta)$ invariant and hence $\left(\Omega^{M}, a \Omega^{j=-M}\right)=0$ if $\Theta(a)=-a, a \in \mathfrak{U}^{P M}$. Therefore $\varphi_{\beta}$ is also $\Theta$-invariant and is determined by its restriction to $\mathfrak{U}_{+}^{P}$, i.e. $\varphi_{\beta}(a)=\varphi_{\beta}\left(a_{+}\right), a_{+}=(a+\Theta(a)) / 2 \in \mathfrak{U}_{+}^{P}$ for any $a \in \mathfrak{U}^{P}$. Therefore we obtain the following

Lemma 3.2. $\varphi_{\beta}$ is the unique $\Theta$-invariant extension, to $\mathfrak{A}^{P}$, of a state $\varphi_{\beta}^{+}$on $\mathfrak{A}_{+}^{P}=\mathfrak{A}_{+}^{F}$, where $\varphi_{\beta}^{+}$is the restriction, to $\mathfrak{A}_{+}^{F}$, of the Fock state $\varphi_{E_{-}}$on $\mathfrak{A}^{F}$ and $E_{-}$is the spectral projection of $H$ (given by (3.5)) for $(-\infty, 0)$. 
We now prove Lemma 3.1. The unitary operator $U$ has the spectral decomposition $U=\int_{0}^{2 \pi} e^{i \theta} d E_{U}(\theta)$ with a simple Lebesgue spectral measure on $[0,2 \pi]$, as is well-known from the theory of Fourier series. Thus $H=\int H(\theta) d E_{U}(\theta)$ with $\theta$ dependent $2 \times 2$ matrix $H(\theta)$, given by

$$
\begin{aligned}
2 H(\theta) & =-\gamma(\theta) V(\theta), \\
V(\theta) & =\left(\begin{array}{ll}
\cos \vartheta(\theta) & -i \sin \vartheta(\theta) \\
i \sin \vartheta(\theta) & -\cos \vartheta(\theta)
\end{array}\right),
\end{aligned}
$$

where $\gamma(\theta) \geqq 0$ is determined by

$$
\cosh 2 K_{1}^{*} \cosh 2 K_{2}-\sinh 2 K_{1}^{*} \sinh 2 K_{2} \cos \theta=\cosh \gamma(\theta),
$$

and $\delta(\theta) \equiv \vartheta(\theta)-\theta$ is determined by

$$
\begin{aligned}
& \cos \delta(\theta)=(\sinh \gamma(\theta))^{-1}\left(\cosh 2 K_{1}^{*} \sinh 2 K_{2}-\sinh 2 K_{1}^{*} \cosh 2 K_{2} \cos \theta\right), \\
& \sin \delta(\theta)=(\sinh \gamma(\theta))^{-1} \sinh 2 K_{1}^{*} \sin \theta
\end{aligned}
$$

(See $[14,11,16]$.) The right-hand side of $(3.8)$ is not 1 except for discrete values of $\theta$ satisfying $\cos \theta=1$ (and only if $K_{1}^{*}=K_{2}$ ) due to

$$
\begin{aligned}
& \left|\sinh 2 K_{1}^{*} \sinh 2 K_{2} \cos \theta\right|+1 \leqq \sinh 2 K_{1}^{*} \sinh 2 K_{2}+1 \\
& \quad \leqq\left(\sinh ^{2} 2 K_{1}^{*}+1\right)^{1 / 2}\left(\sinh ^{2} 2 K_{2}+1\right)^{1 / 2}=\cosh 2 K_{1}^{*} \cosh 2 K_{2} .
\end{aligned}
$$

Thus $\delta(\theta)$ is well-defined (modulo $2 \pi$ ) by (3.9) for all $\theta$ if $K_{1}^{*} \neq K_{2}$ and for all $\theta \neq 0$ (modulo $2 \pi$ ) if $K_{1}^{*}=K_{2}$. (If $K_{1}^{*}=K_{2}, \gamma(\theta)=0$ for $\theta=0$ and any value of $\vartheta(0)$ leads to the same $H(0)$.) Since the matrix part of $(3.7)$ is selfadjoint unitary, $H(\theta)$ does not have an eigenvalue 0 .

Lemma 3.3. Let $\lim A_{n}=A, E_{n}$ and $E$ be the spectral projections of $A_{n}$ and $A$ for an (infinite or finite) interval $(b, a)$ either including or not including the eigenprojections of $a$ and/or $b$. If $a$ and $b$ are not eigenvalues of $A$, then

$$
\lim E_{n}=E \text {. }
$$

Proof. It is enough to treat the case of $b=-\infty, a$ finite because the case of $a=\infty, b$ finite will follow by considering $-A_{n} \rightarrow-A$ and the case of $a$ and $b$ finite will follow by taking the product of projections for the two infinite cases.

For any given vector $\Psi$ and $\varepsilon>0$, there exists $\Phi$ belonging to the $A$-spectral subspace for the complement of the interval $(a-\delta, a+\delta)$ for some $\delta>0$ and satisfying $\|\Psi-\Phi\|<\varepsilon$. Since $E_{n}$ is uniformly bounded, it is enough to prove

$$
\lim E_{n} \Phi=E \Phi
$$

for such $\Phi$.

However, on the $A$-spectral subspace for the complement of the interval $(a-\delta$, $a+\delta$ ), we can apply Theorem 2 of [6] to the characteristic function of the interval $(a, b)$ (the endpoints $a$ and/or $b$ included or not included according to the definition of $E_{n}$ ) and obtain (3.12). 


\section{A General Criterion for Triviality of Center}

In this section, we develop a method of deciding whether $\varphi_{\beta}$ is factorial from a property of the projection $E_{-}$.

We consider the following general situation. Let $\mathfrak{A}$ be a unital $C^{*}$-algebra with two automorphisms $\alpha$ and $\beta$ satisfying

$$
\alpha^{2}=\beta^{2}=\mathbf{1}, \quad \alpha \beta=\beta \alpha,
$$

and with a unitary element $U$ satisfying

$$
\alpha(U)=-U
$$

Let $\hat{\mathfrak{A}}$ be the crossed product of $\mathfrak{A}$ by $\beta$ action of $\mathbb{Z}_{2}$, which is generated by $\mathfrak{A}$ and $T \in \hat{\mathfrak{Q}}$ satisfying

$$
T^{2}=1, \quad T^{*}=T, \quad T a=\beta(a) T(a \in \mathfrak{U})
$$

Let

$$
\mathfrak{H}_{ \pm}=\{a \in \mathfrak{U}: \alpha(a)= \pm a\} .
$$

Let

$$
\mathfrak{B}=\mathfrak{A}_{+}+T \mathfrak{U}_{-} .
$$

It is a $C^{*}$-subalgebra of $\hat{\mathfrak{U}}$. (Note that $\mathfrak{U}_{ \pm}$are $\beta$-invariant.) We extend $\alpha$ and $\beta$ to $\hat{\mathfrak{U}}$ by

$$
\begin{aligned}
& \hat{\alpha}(a+T b)=\alpha(a)+T \alpha(b), \\
& \hat{\beta}(a+T b)=\beta(a)+T \beta(b),
\end{aligned}
$$

where $a, b \in \mathfrak{A}$. For an $\alpha$-invariant state $\varphi$ of $\mathfrak{A}$, there corresponds a unique $\hat{\alpha}$ invariant state $\varphi^{\mathfrak{B}}$ of $\mathfrak{B}$ which is an extension of the restriction of $\varphi$ to $\mathfrak{U}_{+}$.

In our application $\mathfrak{U}=\mathfrak{U}^{F}, \alpha=\Theta, \beta=\Theta_{-}, \mathfrak{B}=\mathfrak{U}^{P}, \varphi^{\mathfrak{B}}=\varphi_{\beta}$ and $U=2^{-1 / 2}$ $\left(c_{i}+c_{i}^{*}\right)$ for any fixed $i$. Our claim in the above general situation is the following:

Theorem 2. Assume that $\varphi$ is an $\alpha$-invariant pure state of $\mathfrak{U}$. Then $\varphi^{\mathfrak{B}}$ is not pure if and only if

(1) $\varphi$ and $\varphi \circ \beta$ are equivalent and

(2) $\varphi_{+}=\varphi \mid \mathfrak{U}_{+} \circ \beta$ are not equivalent.

If $\varphi^{\mathfrak{B}}$ is not pure, it is a mixture of two non-equivalent pure states.

The proof is divided into several lemmas.

Lemma 4.1. If $\varphi$ is $\alpha$-invariant and pure, then $\varphi_{+}$is pure.

Proof. Let $p=(\mathbf{1}+\alpha) / 2$. Then $\varphi_{+} \rightarrow \varphi=\varphi_{+} \circ p$ and $\varphi \rightarrow \varphi_{+}=\varphi \mid \mathfrak{U}_{+}$yield a bijective affine map between states $\varphi_{+}$of $\mathfrak{A}_{+}$and $\alpha$-invariant states $\varphi$ of $\mathfrak{A}$. Hence $\varphi_{+}$is pure if and only if $\varphi$ is an extremal $\alpha$-invariant state. In particular, if $\varphi$ is $\alpha$ invariant and pure, then $\varphi_{+}$is pure.

Lemma 4.2. If an $\alpha$-invariant state $\varphi$ of $\mathfrak{A}$ is pure, then $\varphi_{+}$and $\varphi_{-} \equiv \varphi_{+} \circ \operatorname{Ad} U$ are disjoint. (The disjointness refers to the associated cyclic representations of $\mathfrak{A}_{+}$.)

(Note that $U a U^{*} \in \mathfrak{H}_{+}$if $a \in \mathfrak{U}_{+}$due to $\alpha(U)=-U$.) 
Proof. Let $\mathscr{H}_{\varphi}, \pi_{\varphi}$ and $\xi_{\varphi}$ be the (GNS) triplet of a Hilbert space, a representation of $\mathfrak{U}$ and a cyclic vector associated with $\varphi$. Due to $\alpha$-invariance of $\varphi$,

$$
\mathscr{H}_{\varphi}=\mathscr{H}_{+} \oplus \mathscr{H}_{-}, \quad \mathscr{H}_{ \pm}=\overline{\pi_{\varphi}\left(\mathfrak{U}_{ \pm}\right) \xi_{\varphi}} .
$$

Then $\mathscr{H}_{\varphi_{ \pm}}, \pi_{\varphi_{ \pm}}$and $\xi_{\varphi_{ \pm}}$can be identified with $\mathscr{H}_{ \pm}, \pi_{ \pm}=\left(\pi_{\varphi} \mid \mathfrak{U}_{+}\right) \mid \mathscr{H}_{ \pm}$and $\xi_{+}=\xi_{\varphi}$, $\xi_{-}=\pi_{\varphi}(U) \xi_{\varphi}$. By Lemma 3.1, $\varphi_{+}$and hence $\varphi_{-}=\varphi_{+} \circ$ Ad $U$ are pure. Therefore $\pi_{ \pm}$ are both irreducible. We now derive a contradiction assuming that they are equivalent.

Since $\pi_{ \pm}$are irreducible, there exists a unitary $W_{0} \in \pi_{+}\left(\mathfrak{U}_{+}\right)^{\prime \prime}, \operatorname{Ad} W_{0}$ on $\pi_{+}\left(\mathfrak{U}_{+}\right)$ implementing Ad $U$ on $\mathfrak{U}_{+}$. Since $\pi_{+} \sim \pi_{-}$, there exists a unitary $W \in \pi_{\varphi}\left(\mathfrak{U}_{+}\right)^{\prime \prime}, \mathrm{Ad} W$ on $\pi_{\varphi}\left(\mathfrak{A}_{+}\right)$implementing Ad $U$ on $\mathfrak{U}_{+}$. Since (Ad $\left.W\right) W=W, W$ has to commute with $\pi_{\varphi}(U)$. For $a \in \mathfrak{A}_{-}, a U^{*} \in \mathfrak{A}_{+}$, and

$$
\begin{aligned}
(\operatorname{Ad} W) \pi_{\varphi}(a) & =(\operatorname{Ad} W)\left(\pi_{\varphi}\left(a U^{*}\right) \pi_{\varphi}(U)\right) \\
& =\left\{(A d W) \pi_{\varphi}\left(a U^{*}\right)\right\} \pi_{\varphi}(U) \\
& =\pi_{\varphi}\left(\left\{(\operatorname{Ad} U)\left(a U^{*}\right)\right\} U\right)=\pi_{\varphi}((\operatorname{Ad} U) a) .
\end{aligned}
$$

Therefore Ad $W$ coincides with $\operatorname{Ad} \pi_{\varphi}(U)$ on $\pi_{\varphi}(\mathfrak{U})$ and hence on $\pi_{\varphi}(\mathfrak{Q})^{\prime \prime}$. Since $\pi_{\varphi}$ is irreducible, $W=c \pi_{\varphi}(U)$ for some complex number $c$ of modulus 1 .

On the other hand, by $\alpha$-invariance of $\varphi, \alpha$ can be extended to an isomorphism $\bar{\alpha}$ of $\pi_{\varphi}(\mathfrak{A})^{\prime \prime}$. By $W \in \pi_{\varphi}\left(\mathfrak{U}_{+}\right)^{\prime \prime}$, we have $\bar{\alpha}(W)=W$, whilst $\alpha(U)=-U$. This contradicts with $W=c \pi_{\varphi}(U), W^{*} W=1$.

Lemma 4.3. Let $\varphi$ be an $\alpha$-invariant pure state of $\mathfrak{U}$. The states $\varphi_{+}=\varphi \mid \mathfrak{H}_{+}$and $\varphi_{+} \circ \beta \circ \operatorname{Ad} U$ of $\mathfrak{U}_{+}$are equivalent if and only if

(1) $\varphi$ is equivalent to $\varphi \circ \beta$ and

(2) $\varphi_{+}$is not equivalent to $\varphi_{+} \circ \beta$.

Proof. If $\varphi_{+} \sim \varphi_{+} \circ \beta \circ \operatorname{Ad} U$, then states $\varphi=\varphi_{+}^{\circ} p$ and $\varphi \circ \beta \circ \operatorname{Ad} U=$ $\varphi_{+} \circ \beta \circ \mathrm{Ad} U \circ p$ of $\mathfrak{A}$ are equivalent due to a result of Stratila and Voiculescu (Sect. 2.7 of [17]). Since Ad $U$ is inner on $\mathfrak{A}$, we obtain $\varphi \sim{ }^{\prime} \varphi \circ \beta$. (Note that $[\alpha, \operatorname{Ad} U]=0$ due to $\alpha(U)=-U$.)

Now assume that $\varphi \sim \varphi \circ \beta$. Since $\pi_{\varphi} \mid \mathfrak{U}_{+}=\pi_{+} \oplus \pi_{-}$and $\pi_{ \pm}$are disjoint by Lemmas 4.1 and 4.2, we have two alternatives:

(A) $\pi_{+} \sim \pi_{+} \circ \beta$ disjoint from $\pi_{-} \sim \pi_{-} \circ \beta$,

(B) $\pi_{+} \sim \pi_{-} \circ \beta$ disjoint from $\pi_{-} \sim \pi_{+} \circ \beta$.

The desired conclusion $\pi_{+} \sim \pi_{-} \circ \beta\left(\sim \pi_{+} \circ \beta \circ \operatorname{Ad} U\right)$ holds in and only in case (B), which is characterized by $\varphi \sim \varphi \circ \beta$ and $\pi_{+}$disjoint from $\pi_{+} \circ \beta$.

Lemma 4.4. If $\varphi_{+}$and $\varphi_{+} \circ \beta \circ \mathrm{Ad} U$ are disjoint, then $\varphi^{\mathfrak{B}}$ is pure.

Proof. Let $\mathscr{H}_{\varphi}^{\beta}, \pi_{\varphi}^{\beta}$ and $\xi_{\varphi}^{\beta}$ be the GNS triplet for the state $\varphi \circ \beta$. Let

$$
\begin{gathered}
\hat{\mathscr{H}}_{\varphi}=\mathscr{H}_{\varphi} \oplus \mathscr{H}_{\varphi}^{\beta}, \\
\hat{\pi}_{\varphi}(a+T b)\left[\pi_{\varphi}\left(a_{1}\right) \xi_{\varphi} \oplus \pi_{\varphi}^{\beta}\left(a_{2}\right) \xi_{\varphi}^{\beta}\right] \\
=\pi_{\varphi}\left(a a_{1}+\beta\left(b a_{2}\right)\right) \xi_{\varphi} \oplus \pi_{\varphi}^{\beta}\left(a a_{2}+\beta\left(b a_{1}\right)\right) \xi_{\varphi}^{\beta} .
\end{gathered}
$$


Then the restriction of $\hat{\pi}_{\varphi}(\mathfrak{B})$ to

$$
\mathscr{H}_{\varphi}^{\mathfrak{B}}=\mathscr{H}_{+} \oplus \mathscr{H}_{-}^{\beta} \quad\left(\mathscr{H}_{ \pm}^{\beta} \equiv \overline{\left.\pi_{\varphi}^{\beta}\left(\mathcal{U}_{ \pm}\right) \xi_{\varphi}^{\beta}\right)}\right.
$$

(denoted $\pi_{\varphi}^{\mathfrak{B}}$ ) yields the cyclic representation of $\mathfrak{B}$ associated with $\varphi^{\mathfrak{B}}$.

Since $\pi_{\varphi}^{\mathfrak{B}} \mid \mathfrak{U}_{+} \sim \pi_{+} \oplus \pi_{-} \circ \beta$ and the assumption implies the disjointness of $\pi_{+}$ and $\pi_{-} \circ \beta$ (due to $\pi_{-} \circ \beta=\pi_{+}{ }^{\circ} \operatorname{Ad} U \circ \beta=\pi_{+} \circ \beta \circ \operatorname{Ad} \beta(U) \sim \pi_{+} \circ \beta \circ \operatorname{Ad} U$ because of $U^{*} \beta(U) \in \mathfrak{A}_{+}$, any $X \in \pi_{\varphi}^{\mathfrak{B}}\left(\mathfrak{U}_{+}\right)^{\prime}$ is of the form

$$
X=\lambda \mathbf{1}_{+} \oplus \mu \mathbf{1}_{-, \beta} .
$$

If $X \in \pi_{\varphi}^{\mathfrak{B}}(\mathfrak{Q})^{\prime}, X$ must commute with $\pi_{\varphi}^{\mathfrak{B}}(T U)$, which connects $\mathscr{H}_{+}$with $\mathscr{H}_{-}^{\beta}$ and hence $\lambda=\mu$. This proves that $\pi_{\varphi}^{\mathfrak{B}}$ is irreducible.

Lemma 4.5. If $\varphi_{+}$is equivalent to $\varphi_{+}{ }^{\circ} \beta \circ \mathrm{Ad} U$, then $\varphi^{\mathfrak{B}}$ is a non-trivial mixture of two non-equivalent pure states.

Proof. Since $\pi_{+}$is irreducible and $\varphi_{+} \sim \varphi_{+} \circ \beta \circ \operatorname{Ad} U$, there exists a unitary $W_{0} \in \pi_{+}\left(\mathfrak{A}_{+}\right)^{\prime \prime}, \operatorname{Ad} W_{0}$ on $\pi_{+}\left(\mathfrak{A}_{+}\right)$implementing $\beta \circ \operatorname{Ad} U$ on $\mathfrak{A}_{+}$. By $\pi_{+} \sim \pi_{+} \circ \beta \circ \mathrm{Ad}$ $U$, there exists a unitary $W \in \pi_{\varphi}^{\mathfrak{B}}\left(\mathfrak{A}_{+}\right)^{\prime \prime}$, such that Ad $W$ on $\pi_{\varphi}^{\mathfrak{B}}\left(\mathfrak{A}_{+}\right)$implements $\beta \circ \mathrm{Ad}$ $U$ on $\mathfrak{A}_{+}$. Since $($Ad $W) W=W, W$ has to commute with $\pi_{\varphi}^{\mathfrak{B}}(T U)^{*}$. Let

$$
V=W \pi_{\varphi}^{\mathfrak{B}}(T U)^{*} .
$$

For any $a_{-} \in T \mathfrak{A}_{-}, a_{-}=\left(a_{-} T U\right)(T U)^{*}$ and hence

$$
\begin{aligned}
(\operatorname{Ad} W) \pi_{\varphi}^{\mathfrak{B}}\left(a_{-}\right) & =\left\{(\operatorname{Ad} W) \pi_{\varphi}^{\mathfrak{B}}\left(a_{-} T U\right)\right\} \pi_{\varphi}^{\mathfrak{B}}(T U)^{*} \\
& =\pi_{\varphi}^{\mathfrak{B}}\left(\left\{(\hat{\beta} \circ \operatorname{Ad} U)\left(a_{-} T U\right)\right\}(T U)^{*}\right) \\
& =\pi_{\varphi}^{\mathfrak{B}}\left((\hat{\beta} \circ \operatorname{Ad} U)\left(a_{-}\right)\right),
\end{aligned}
$$

because $(\hat{\beta} \circ \operatorname{Ad} U)(T U)=\hat{\beta}(U T)=\beta(U) T=T U$. Therefore $V$ is in the center $\pi_{\varphi}^{\mathfrak{B}}(\mathfrak{B})^{\prime} \cap \pi_{\varphi}^{\mathfrak{B}}(\mathfrak{B})^{\prime \prime}$. It is non-trivial because it connects $\mathscr{H}_{+}$and $\mathscr{H}_{+}^{\beta}$. Thus $\varphi^{\mathfrak{B}}$ is not pure.

Since the restriction of $\pi_{\varphi}^{\mathfrak{B}}$ to $\mathfrak{A}_{+}$is a sum of two equivalent irreducible representations, $\pi_{\varphi}^{\mathfrak{B}}\left(\mathfrak{U}_{+}\right)^{\prime}$ is isomorphic to the algebra of $2 \times 2$ matrices and $\pi_{\varphi}^{\mathfrak{B}}(\mathfrak{B})^{\prime}$ has to be its non-trivial proper $*$-subalgebra, which must be a two-dimensional commutative algebra, coinciding with the center of $\pi_{\varphi}^{\mathfrak{B}}(\mathfrak{B})^{\prime \prime}$. Then $\varphi^{\mathfrak{B}}$ is a non-trivial mixture of two non-equivalent pure states.

\section{Application of the General Criterion}

For $\varphi_{\beta}$ discussed in Sect. 3, which is a Fock state $\varphi_{E}$, the state $\varphi_{\beta} \circ \Theta_{-}$is the Fock state $\varphi_{\theta_{-} E_{-} \theta_{-}}$, where

$$
(\theta-f)_{j}=\left\{\begin{array}{rr}
f_{j} & j \geqq 1 \\
-f_{j} & j \leqq 0 .
\end{array}\right.
$$

By a general criterion for two Fock states to be equivalent (for example, see Theorem 1 of [3]), the condition (1) of Theorem 2 is satisfied if and only if $E_{-}-\theta_{-} E_{-} \theta_{-}$is in 
the Hilbert-Schmidt class. We have

$$
\begin{aligned}
& \left\|E_{-}-\theta_{-} E_{-} \theta_{-}\right\|_{\mathrm{HS}}^{2}=\operatorname{tr}\left(\left(\mathbf{1}-E_{-}\right) \theta_{-} E_{-} \theta_{-}\left(\mathbf{1}-E_{-}\right)\right. \\
& \left.\quad+E_{-} \theta_{-}\left(\mathbf{1}-E_{-}\right) \theta_{-} E_{-}\right)=\left\|E_{-} \theta_{-}\left(\mathbf{1}-E_{-}\right)\right\|_{\mathrm{HS}}^{2} \\
& \quad+\left\|\left(\mathbf{1}-E_{-}\right) \theta_{-} E_{-}\right\|_{\mathrm{HS}}^{2}=2\left\|E_{-} \theta_{-}\left(\mathbf{1}-E_{-}\right)\right\|_{\mathrm{HS}}^{2},
\end{aligned}
$$

where the last equality is due to $\Gamma \theta_{-} \Gamma=\theta_{-}$and $\Gamma E_{-} \Gamma=\left(1-E_{-}\right)$. By evaluating this quantity in Sect. 6, we obtain the following:

Lemma 5.1. $\varphi_{E_{-}}$and $\varphi_{\theta_{-} E_{-} \theta_{-}}$are equivalent if $\beta \neq \beta_{c}$. They are not equivalent if $\beta=\beta_{c}$.

To deal with the second condition of Theorem 2 , we introduce the following $\mathbb{Z}_{2}$ index between two basis projections $E_{1}$ and $E_{2}$ for which $E_{1}-E_{2}$ is in the HilbertSchmidt class:

$$
\sigma\left(E_{1}, E_{2}\right)=(-1)^{\operatorname{dim} E_{1} \wedge\left(1-E_{2}\right)} .
$$

By $\Gamma\left\{E_{1} \wedge\left(\mathbf{1}-E_{2}\right)\right\} \Gamma=\left(\mathbf{1}-E_{1}\right) \wedge E_{2}, \sigma$ is symmetric in $E_{1}$ and $E_{2}$. We prove the following in Sect. 7:

Theorem 3. $\sigma\left(E_{1}, E_{2}\right)$ is continuous in $E_{1}$ and $E_{2}$ with respect to the norm topology of E's.

The criterion for the equivalence of the restriction of Fock states to $\mathfrak{A}_{+}$is given by the following theorem to be proved in Sect. 7 .

Theorem 4. The restrictions of Fock states $\varphi_{E_{1}}$ and $\varphi_{E_{2}}$ of $\mathfrak{A}^{F}$ to the even part $\mathfrak{U}_{+}^{F}$ are equivalent if and only if

(i) $E_{1}-E_{2}$ is in the Hilbert-Schmidt class and

(ii) $\sigma\left(E_{1}, E_{2}\right)=1$.

We shall show the following result in Sect. 6 using this criterion.

Lemma 5.2. $\sigma\left(E_{-}, \theta_{-} E_{-} \theta_{-}\right)$is 1 if $\beta<\beta_{c}$ and -1 if $\beta>\beta_{c}$.

Proof of this lemma in Sect. 6 consists of two parts, one proving the norm continuity of $E_{-}$in the positive parameters $K_{1}^{*}$ and $K_{2}$ as long as $K_{1}^{*} \neq K_{2}$. Theorem 3 then implies the constancy of $\sigma\left(E_{-}, \theta_{-} E_{-} \theta_{-}\right)$for $K_{2}<K_{1}^{*}$ and for $K_{1}^{*}<K_{2}$. Finally $\sigma\left(E_{1}, E_{2}\right)=1$ is explicitly shown for $K_{2}=0$ and $\sigma\left(E_{1}, E_{2}\right)=-1$ for $K_{1}^{*}=0$. $\left(\beta \gtrless \beta_{c}\right.$ if and only if $K_{2} \gtrless K_{1}^{*}$.)

Theorem 2, Theorem 4, Lemma 5.1 and Lemma 5.2 imply Theorem 1.

\section{Equivalence of $\varphi_{\beta}$ and $\varphi_{\beta}{ }^{\circ} \Theta_{-}$(Proof of Lemma 5.1)}

(1) Equivalence for $K_{1}^{*} \neq K_{2}$

Denoting $q_{ \pm}=\left(\mathbf{1} \pm \theta_{-}\right) / 2$, we have

$$
\begin{aligned}
& \left\|E_{-} \theta_{-}\left(1-E_{-}\right)\right\|_{\mathrm{HS}}^{2}=4\left\|\left(1-E_{-}\right) q_{+} E_{-}\right\|_{\mathrm{HS}}^{2} \\
& \quad=4 \lim _{\varepsilon \rightarrow 0} \int_{-\pi}^{\pi} \frac{d \theta_{1}}{2 \pi} \int_{-\pi}^{\pi} \frac{d \theta_{2}}{2 \pi}\left|1-e^{i\left(\theta_{1}-\theta_{2}\right)-\varepsilon}\right|^{-2} \operatorname{tr}\left(E_{-}\left(\theta_{1}\right) E_{+}\left(\theta_{2}\right)\right),
\end{aligned}
$$


where $E_{ \pm}(\theta)=(1 \pm V(\theta)) / 2$ are eigenprojections of (3.7b) belonging to eigenvalues \pm 1 and

$$
\begin{aligned}
\operatorname{tr}\left(E_{-}\left(\theta_{1}\right) E_{+}\left(\theta_{2}\right)\right) & =\left\{2-\operatorname{tr} V\left(\theta_{1}\right) V\left(\theta_{2}\right)\right\} / 4 \\
& =\left\{1-\cos \left(\vartheta\left(\theta_{1}\right)-\vartheta\left(\theta_{2}\right)\right)\right\} / 2 .
\end{aligned}
$$

If $K_{1}^{*} \neq K_{2}$, then $\gamma(\theta)$ defined by (3.8) is a strictly positive, real analytic function of $\theta$ with a period $2 \pi$ due to the strict inequality in (3.10) for $K_{1}^{*} \neq K_{2}$. Therefore $\cos \vartheta(\theta)$ and $\sin \vartheta(\theta)$ are real analytic functions of $\theta$ with a period $2 \pi$ due to (3.9) and $\vartheta(\theta)=$ $\theta+\delta(\theta)$. Therefore the integrand of (6.1) is uniformly bounded even at $\varepsilon=0$ and (6.1) is finite.

(2) Non-equivalence for $K_{1}^{*} \equiv K_{2} \equiv K$

For $\cos \theta \neq 1$ (i.e. $\theta \neq 0 \bmod 2 \pi$ ), $\cos \vartheta(\theta)$ and $\sin \vartheta(\theta)$ are real analytic in $\theta$, and hence the integrand of (6.1) is integrable except possibly near the point $\theta_{1}=\theta_{2}=0$. As $\theta \rightarrow 0, \gamma(\theta) \rightarrow+0$ with

$$
\lim _{\theta \rightarrow 0}(\gamma(\theta) / \theta)^{2}=\sinh ^{2} 2 K
$$

Therefore $\delta(\theta) \rightarrow \pm \pi / 2(\bmod 2 \pi)$ as $\theta \rightarrow \pm 0$ by (3.9). Thus the contribution from $\theta_{1} \theta_{2}>0$ in (6.1) in a neighbourhood of $\theta_{1}=\theta_{2}=0$ is finite while the contribution from $\theta_{1} \theta_{2}<0$ is $+\infty$ due to

$$
\int_{0}^{\alpha} d \theta_{1} \int_{-\alpha}^{0} d \theta_{2}\left\{\left(1-\cos \left(\theta_{1}-\theta_{2}\right)\right)^{2}+\sin ^{2}\left(\theta_{1}-\theta_{2}\right)\right\}^{-1}=\infty .
$$

Therefore $\varphi_{\beta}$ and $\varphi_{\beta} \circ \Theta_{-}$are disjoint at $\beta=\beta_{c}$.

(3) We now compute the $\mathbb{Z}_{2}$ index. We consider $K_{1}^{*}$ and $K_{2}$ as parameters. For $K_{1}^{*} \neq K_{2}, \varphi_{\beta}$ and $\varphi_{\beta} \circ \Theta_{-}$are equivalent.

Furthermore the continuity of $E_{-}$on the parameters $K_{1}^{*}$ and $K_{2}$ in the region $K_{1}^{*} \neq K_{2}$ can be seen as follows.

Let the functions $\delta, \vartheta, V$ and the projections $E_{+}$for another parameter $K_{1}^{\prime *}$ and $K_{2}^{\prime}$ be denoted by $\delta^{\prime}, \vartheta^{\prime}, V^{\prime}$ and $E_{ \pm}^{\prime}$. Then

$$
\begin{aligned}
\left\|E_{-}-E_{-}^{\prime}\right\| & =\sup _{\theta}\left\|(\mathbf{1}-V(\theta))-\left(\mathbf{1}-V^{\prime}(\theta)\right)\right\| / 2 \\
& =2^{-1} \sup _{\theta}\left\|V(\theta)-V^{\prime}(\theta)\right\| .
\end{aligned}
$$

For $K_{1}^{*} \neq K_{2}, \gamma(\theta)$ is real analytic in $K_{1}^{*}, K_{2}$ and $\theta$ by (3.8) and so are $\cos \delta(\theta)$ and $\sin \delta(\theta)$ due to $\gamma(\theta) \neq 0$. Hence $V(\theta)$ is uniformly continuous in $K_{1}^{*}, K_{2}$ and $\theta$ over a compact set. In particular, (6.5) tends to 0 as $\left(K_{1}^{\prime *}, K_{2}^{\prime}\right)$ tends to $\left(K_{1}^{*}, K_{2}\right)$, and hence $E_{-}$is continuous in the parameter $K_{1}^{*}$ and $K_{2}$ relative to the norm topology except for $K_{1}^{*}=K_{2}$.

Hence, by Theorem 3, we have only to find the $\mathbb{Z}_{2}$-index for (A) $K_{2}=0, K_{1}^{*}>0$ and (B) $K_{1}^{*}=0, K_{2}>0$.

Case (A). From (3.8) and (3.9), we have $\gamma(\theta)=2 K_{1}^{*}, \delta(\theta)=\pi-\theta, \vartheta(0)=\pi, V(\theta)=$ 
$\left(\begin{array}{rr}-1 & 0 \\ 0 & 1\end{array}\right) \cdot\left(\right.$ Or $H=K_{1}^{*} H_{1}$ by (3.5).) Therefore $E_{-}=\theta_{-} E_{-} \theta_{-}$and $\sigma\left(E_{-}, \theta_{-} E_{-} \theta_{-}\right)$ $=1$ for $K_{2}=0$ and hence for $K_{1}^{*}>K_{2}$.

Case (B). We have $\mathrm{H}=\mathrm{K}_{2} \mathrm{H}_{2}$ with the selfadjoint unitary $H_{2}$ given by (2.16). By $E_{ \pm}=\left(\mathbf{1} \pm H_{2}\right) / 2, h=\left(\begin{array}{c}f \\ g\end{array}\right) \in\left(E_{-} \wedge \theta_{-}\left(\mathbf{1}-E_{-}\right) \theta_{-}\right) \mathscr{K}$ has to satisfy

$$
\begin{aligned}
2\left(1-E_{-}\right) h & =\left(\mathbf{1}+H_{2}\right) h=0, \\
2 \theta_{-}\left(\theta_{-} E_{-} \theta_{-}\right) h & =\left(\mathbf{1}-H_{2}\right) \theta_{-} h=0 .
\end{aligned}
$$

To solve this simultaneous equation, let $\left(\begin{array}{l}f_{1} \\ g_{1}\end{array}\right) \equiv h_{1}=v h$ with $v=2^{-1 / 2}$ $\left(\begin{array}{rr}1 & 1 \\ 1 & -1\end{array}\right)$. Since $v^{2}=1$ and

we obtain

$$
v H_{2} v=-\left(\begin{array}{ll}
0 & U \\
U^{*} & 0
\end{array}\right)
$$

$$
\begin{aligned}
U g_{1} & =f_{1}, U^{*} f_{1}=g_{1}, \\
U \theta_{-} g_{1} & =-\theta_{-} f_{1}, U^{*} \theta_{-} f_{1}=-\theta_{-} g_{1} .
\end{aligned}
$$

In particular, we have

$$
\left(U+\theta_{-} U \theta_{-}\right) g_{1}=0,\left(U^{*}+\theta_{-} U^{*} \theta_{-}\right) f_{1}=0 .
$$

These equations mean that all components of $g_{1}$ except for the $n=1$ component and all components of $f_{1}$ except for the $n=0$ component vanish. Furthermore, the $n=1$ component of $g_{1}$ and the $n=0$ component of $f_{1}$ are equal. Conversely such $f_{1}$ and $g_{1}$ satisfy (6.9) and (6.10). Therefore

$$
\operatorname{dim}\left(E_{-} \wedge \theta_{-}\left(1-E_{-}\right) \theta_{-}\right)=1 .
$$

Hence $\sigma\left(E_{-}, \theta_{-} E_{-} \theta_{-}\right)=-1$ for $K_{1}^{*}<K_{2}$.

\section{Equivalence Criterion for the Fock Representation of the Even Part $\mathfrak{U}_{+}^{F}$}

Proof of Theorem 4. Let $\varphi_{1+}$ and $\varphi_{2+}$ denote the restrictions of $\varphi_{E_{1}}$ and $\varphi_{E_{2}}$ to $\mathfrak{A}_{+}^{F}$. If $\varphi_{1+}$ and $\varphi_{2+}$ are equivalent, then $\varphi_{E_{1}}$ and $\varphi_{E_{2}}$ must be equivalent due to a result of Stratila and Voiculescu (Sect. 2.7 of [17]). In view of Theorem 1 of [3], this implies that the condition (i) is a necessary condition.

We now assume that (i) holds. We follow the proof of Lemma 9.4 in [3]. There exists a Bogoliubov transformation $U$ which does not change $E_{j}\left(\mathbf{1}-E_{\pi / 2}\right)(j=1,2)$ and changes $E_{2} E_{\pi / 2}$ to $E_{1} E_{\pi / 2}$, where

$$
E_{\pi / 2}=\left\{E_{1} \wedge\left(1-E_{2}\right)\right\}+\left\{\left(1-E_{1}\right) \wedge E_{2}\right\},
$$

namely

$$
E_{2}^{\prime} \equiv U^{*} E_{2} U=E_{2}\left(1-E_{\pi / 2}\right)+E_{1} E_{\pi / 2} .
$$


The Bogoliubov automorphism of $\mathfrak{A}^{F}$ induced by this $U$ is inner and the implementing unitary operator $\hat{Q}(U)$ belongs to $\mathfrak{U}_{\sigma}^{F}, \sigma=+$ or - according to whether the $\mathbb{Z}_{2}$-index (5.3) is even or odd. Under the condition (i), there is another Bogoliubov transformation $R\left(E_{2}^{\prime} / E_{1}\right)$, which brings $E_{2}^{\prime}$ to $E_{1}$

$$
R\left(E_{2}^{\prime} / E_{1}\right) * E_{2}^{\prime} R\left(E_{2}^{\prime} / E_{1}\right)=E_{1},
$$

and which is implemented in the Fock representation $\pi_{1}$ associated with $\varphi_{E_{1}}$ by a unitary operator $Q\left(R\left(E_{2}^{\prime} / E_{1}\right)\right)$ in $\pi_{1}\left(\mathfrak{A}_{+}^{F}\right)^{\prime \prime}$. (In the proof of Lemma 9.4 of [3], it is implemented by a limit of a complex multiple of unitary elements $Q\left(g_{n}\right)$, in the notation of Lemma 9.3, which is of a form $\pi_{1}(\exp i(B, H B) / 2)$ according to Sect. 8 of [3] and belongs to $\pi_{1}\left(\mathfrak{U}_{+}^{F}\right)$.) Therefore the cyclic representation $\pi_{2+}$ associated with $\varphi_{2+}$ (which is the restriction of the cyclic representation $\pi_{2}$ of $\mathfrak{U}^{F}$ associated with $\varphi_{E_{2}}$ to the subalgebra $\mathfrak{A}_{+}^{F}$ and to the subspace $\left(\mathfrak{U}_{+}^{F} \xi_{2}\right)$, where $\xi_{2}$ is the cyclic vector for $\left.\varphi_{E_{2}}\right)$ is equivalent to the representation $\pi_{1+}$ or $\pi_{1-}$ through the unitary operator $Q\left(R\left(E_{2}^{\prime} / E_{1}\right)\right) \pi_{1}(\hat{Q}(U))$ according to whether the $\mathbb{Z}_{2}$-index is +1 or -1 , where $\pi_{1 \pm}$ are the restrictions of $\pi_{1} \mid \mathfrak{U}_{+}^{F}$ to the closure of $\pi_{1}\left(\mathfrak{U}_{ \pm}^{F}\right) \xi_{1}, \xi_{1}$ being the cyclic vector for $\varphi_{E_{1}}$. In view of Lemma 4.2, $\pi_{2+}$ is equivalent to or disjoint from $\pi_{1+}$ according to whether the $\mathbb{Z}_{2}$-index is +1 or -1 .

Proof of Theorem 3. $E_{1} \wedge\left(1-E_{2}\right)$ is the eigenprojection of $E_{1}-E_{2}$ belonging to the eigenvalue 1 . Let $E_{1}^{0}-E_{2}^{0}$ be in the Hilbert-Schmidt class and $4 \varepsilon(>0)$ be the distance of 1 from $\left\{\operatorname{Spec}\left(E_{1}^{0}-E_{2}^{0}\right)\right\} \backslash\{1\}$. Then there exists a neighbourhood $\mathscr{N}$ of $\left(E_{1}^{0}, E_{2}^{0}\right)$ such that for any $\left(E_{1}, E_{2}\right) \in \mathcal{N}, \operatorname{Spec}\left(E_{1}-E_{2}\right) \subset[-1,1-3 \varepsilon] \cup[1-\varepsilon, 1]$. Then

$$
E=(2 \pi i)^{-1} \int_{|Z-1|=2 \varepsilon}\left(Z-\left(E_{1}-E_{2}\right)\right)^{-1} d Z
$$

must be the spectral projection of $E_{1}-E_{2}$ for the interval $[1-\varepsilon, 1]$. Since it is of finite dimension for $\left(E_{1}^{0}, E_{2}^{0}\right)$ and is continuous in the norm topology by (7.4), $\operatorname{dim} E$ is finite and independent of $\left(E_{1}, E_{2}\right) \in \mathscr{N}$.

Let $\left(E_{1}, E_{2}\right) \in \mathscr{N}$ be a pair of basis projections (i.e. $\left.\Gamma E_{j} \Gamma=1-E_{j}\right)$, such that $E_{1}-$ $E_{2}$ is in the Hilbert-Schmidt class. Theorem 11 of [3] says that the von Neumann algebra generated by $E_{1}$ and $E_{2}$, restricted to an eigensubspace of $\left|E_{1}-E_{2}\right|$ belonging to an eigenvalue not equal to 0 or 1 , is isomorphic to the algebra of all $4 \times 4$ matrices. This means that the multiplicity of such an eigenvalue of $\left|E_{1}-E_{2}\right|$ must be a multiple of 4 . Since $\Gamma\left(E_{1}-E_{2}\right) \Gamma=\left(1-E_{1}\right)-\left(1-E_{2}\right)=-\left(E_{1}-E_{2}\right)$, the multiplicity of an eigenvalue of $E_{1}-E_{2}$ belonging to an eigenvalue not equal to 0 or \pm 1 must be even. Due to the constancy of the dimension of $E$, the multiplicity of the eigenvalue 1 of $E_{1}-E_{2}$ is even or odd according to whether the multiplicity of the eigenvalue 1 of $E_{1}^{0}-E_{2}^{0}$ is even or odd. Namely we have the constancy of the $\mathbb{Z}_{2}$ index $\sigma\left(E_{1}, E_{2}\right)=\sigma\left(E_{1}^{0}, E_{2}^{0}\right)$ in $N$.

Acknowledgements. The second named author would like to thank H. Araki and RIMS, Kyoto University for their hospitality and the Royal Society for their financial support during the period of this work. 


\section{References}

1. Aizenman, M. : Translation invariance and instability of phase coexistence in the two dimensional Ising system. Commun. Math. Phys. 73, 83-94 (1980)

2. Araki, H. : Gibbs states of a one dimensional quantum lattice. Commun. Math. Phys. 14, 120-157 (1969)

3. Araki, H. : On quasifree states of CAR and Bogoliubov automorphisms. Publ. RIMS, Kyoto Univ. 6, 384-442 (1970)

4. Araki, H. : On the XY-model on two sided infinite chain. Preprint RIMS 1983

5. Higuchi, Y.: On the absence of non-translationally invariant Gibbs states for the two-dimensional Ising model. In: Random Fields, Colloquia Societatis Janos Bolyai, 27, Esztergom, Hungary, 1979

6. Kaplansky, I. : A theorem on rings of operators. Pac. J. Math. 1, 227-232 (1951)

7. Kaufman, B. : Crystal Statistics II. Phys. Rev. 76, 1232-1243 (1949)

8. Kaufman, B., Onsager, L. : Crystal Statistics III. Phys. Rev. 76, 1244-1252 (1949)

9. Kramers, H. A., and Wannier, G. H. : Statistics of the two dimensional ferromagnet. Part 1. Phys. Rev. 60, 252-262 (1941)

10. Kuik, R. : Doctoraals Dissertation, Gröningen, 1981

11. Lewis, J. T. Sisson, P. N. M. : A $C^{*}$-algebra of the two dimensional Ising model. Commun. Math. Phys. 44, 279-292 (1975)

12. Lewis, J. T., Winnink, M.: The Ising-model phase transition and the index of states on the Clifford algebra. In: Random fields, Colloquia Societatis Janos Bolyai, 27, Esztergom, Hungary, 1979

13. Onsager, L. : Crystal Statistics I. Phys. Rev. 65, 117-149 (1944)

14. Pirogov, S.: States associated with the two dimensional Ising model. Theor. Math. Phys. 11, 614-617 (1972)

15. Schultz, T. D., Mattis, D. C., Lieb, E. : Two dimensional Ising model as a soluble problem of many Fermions. Rev. Mod. Phys. 36, 856-871 (1964)

16. Sisson, P. N. M. : Ph. D. Thesis, Dublin University, 1974

17. Stratila, S., Voiculescu, D. : On a class of KMS states for the unitary group $\mathfrak{A}(\infty)$. Math. Ann. 235, 87-110 (1978)

Communicated by H. Araki

Received April 7, 1983; in revised form May 31, 1983 
